\title{
A Very Rare Origin of the Left Vertebral Artery and its Clinical Implications
}

\author{
Olutayo Ariyo* \\ Dept. of Anatomy Pathology and Cell Biology, SKMC at Thomas Jefferson University, Philadelphia, USA \\ *Corresponding Author: Olutayo Ariyo, Dept. of Anatomy Pathology and Cell Biology, SKMC at \\ Thomas Jefferson University, Philadelphia, USA, E-mail: Olutayo.Ariyo@jefferson.edu
}

\begin{abstract}
Most variants of the left vertebral artery tend to occursupra-aortic, usually between the left common carotid and the left subclavian arteries. We report a rare variant of the left vertebral artery arising as the most distal and inferior branch off the aortic arch in a 69 year-old male cadaver. Arising posteroinferiorly from the arch, the variant coursed superiorly and medial-ward, posterior to the left subclavian artery, enteringthe transverse cervical foramina at C5 level to run more cranially cervical foramina C5-C2. The variant artery was an observed with some tortuosity just proximal to entry into C5 foramina. The normally arising left or right vertebral artery plays a vital role in the Subclavian Steal Syndrome, a retrograde flow in the ipsilateral vertebral artery in an occlusion proximal origin of its ipsilateral subclavian artery. In our reported variant, modelled with a possible occlusion in the proximal segment of the left subclavian artery, despite an hypothesized retrograde flow in the left vertebral artery will not be helpful in delivering blood into the subclavian-axillary continuum, as such retrograde flow will dump into the aortic arch directly and unhelpful to the occluded left subclavian artery.
\end{abstract}

Knowledge of origin, course and morphology of the vertebral artery is of importance to neurosurgeons, neurovascular interventionists, with regards to selection of appropriate supra-aortic endovascular procedures to help in minimizing dangers of inadvertent iatrogenic lesioning of an aberrant coursing vertebral artery and in assisting neuroradiologists in the correct interpretation of images.

Keywords: Variant left vertebral artery, occlusion proximal origin of left subclavian artery, retrograde flow in ipsilateral vertebral artery, Subclavian Steal Syndrome (SSS), foraminal entry of vertebral artery.

\section{INTRODUCTION}

The usual origins of the vertebral arteries are from thepostero-superior surfaces of their ipsilateral subclavian artery, the artery coursing superiorly medial-ward, usually to enter the transverse foramen of the 6th cervical vertebrae.

The artery is typically divided into 4 segments V1 (preforaminal): origin to transverse foramen of $\mathrm{C} 6, \mathrm{~V} 2$ (foraminal): from the transverse foramen of $\mathrm{C} \mathrm{VI}$ to the transverse foramen of $\mathrm{C} 2$, V3 (atlantic, extradural or extraspinal): from $\mathrm{C} 2$ to the dura and V4 (intradural or intracranial): from the dura to their confluence to form the basilar artery.

The 2 vertebral arteries join to form a single midline basilar artery in the hind brain. Even though there is variability in the origins of the left vertebral artery (LVA) along the aortic arch, however, it had been reported that about $5 \%$ of the left vertebral artery (LVA) will have a variable origin, most usually between the left common carotid artery (LCCA) and the left subclavian artery (LSA). Jayanthi $\mathrm{V}$ et al. (2010) reported an anomalous origin of the LVA from the arch of the aorta [1].

Gluncic V et al, (1999) described case of an unusual origin of both vertebral arteries in a singular cadaver. On the left, the artery arose directly from the common trunk of vertebral and subclavian artery at the aortic arch and enters the transverse cervical foramina at C VI. The right vertebral artery (RVA) on the other hand originated from the right common carotid artery (RCCA) and enters the transverse foramina at $\mathrm{C}$ III [2].

LVA arising as the most distal of the branches off the aortic arch is very rare compared with the one originating between the LCCA and the LSA? Case D et al., (2015) however presented a case report of an anomalous origin of the RVA from the aortic arch distal to the LSA along with a review of cases reported to date in the literature [3]. 
Einstein EH et al, (2016) reported the case of an anomalous origin of the LVA from the aortic arch, arising between the LCCA and the LSA [4]. Shiva Kumar GL, Pamidi N, Somayaji SN, Nayak S, Vollala VR (2010) reported an anomalous branching pattern of the aortic arch, reporting the LCCA arising from the $\mathrm{BCT}$, and a trifurcated brachiocephalic (BCT) with the RVA arising between the LSA and RCCA [5]. Of all cases, vertebral arteries that arose from the aortic arch were much more likely to not only have a more medial course (especially their preforaminal segment) over the cervical vertebra bodies but also to enter a transverse foramen that was more cranially located than the normal C6.

\section{CASE REPORT}

During routine dissection of the thoracic region in our facility, we observed an aortic arch branching variation with four arising vessels arising from the arch, the BCT as the first branch followed by the LCCA. There was no observed truncus bicaroticus morphology as the BCT and LCCA arose independently off the arch. The LCCA was followed by the LSA, but on careful inspection of the LSA, we did not observe the usually arising left vertebral artery from the $s$ supero-posterior surface of the LSA. However following a more careful search, we observed the LVA arising poster inferiorly from the aortic arch distal to the LSA as the last terminal branch, resulting in 4- vessel aortic arch morphology (Fig). The observed LVA coursed superiorly in the mediastinum, travelling medial-ward to run posterior to the LSA. We observed tortuosity in the morphology of the LVA just proximal to the LVA entering the cervical foramina at the level of C5 to continue its cranial-ward cervical course in the more superior cervical foramina (Fig1). We did not observe any other abnormality distal to the LVA.

The LVA as the last branch off the aortic arch was carefully examined to ensure it is not an aberrant right subclavian artery (RSA) another supra aortic arising variant vessel sometimes reported as the last branch off the aortic arch. There were no other abnormalities observed along the aortic arch. The BCT arose normally as the first branch, bifurcating to yield the RSA and the RCCA. The RVA originated normally from the posterosuprior surface of the RSA. The LCCA also originated independently off the aortic arch, without the presence of a bovine morphology (Fig1).

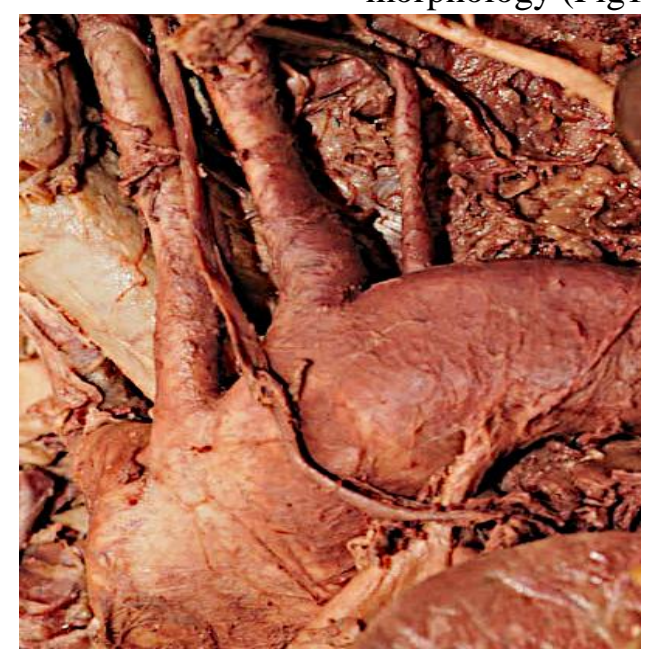

Fig.1. The image shows the left vertebral artery arising as the last vessel off the aortic arch, arising distal to the left subclavian artery. LVA; left vertebral artery, LCCA; left common carotid artery, LSA; left subclavian artery, BCT; brachiocephalic trunk.

\section{DisCUSSIONS}

The most common anomaly of the usual aortic arch is the direct LVA origin off the aortic arch between the LCCA and the LSA, converting the arch into four vessel morphology. Such a variant has been widely reported in many surgical, radiologic, post- mortem and cadaveric studies, the reports are either incidental or in large sample studies. Or as incidental findings, with a Bhatia K, et al., (2005) reported a $7.41 \%$ incidence [6] while Budhiraja V et al., (2013) reported a much higher $15.3 \%$ incidence of the LVA arising from the arch between the LCCA and the LSA [7].

TheLVA arising from the aortic arch is one of the aberrant supraaortic branching variations. Other supra-aortic variants include the LCCA sharing a common trunk with the BCT (the bovine arch morphology), the LCCA arising from the $\mathrm{BCT}$, an aberrant RSA originating 
from the aortic arch as the last branch distal to the LSA and the LVA sharing a common stem of origin with the LSA directly off the aortic arch. Nelson ML, Sparks CD (2001) reported an unusual instance of the four arteries arising from the arch of the aorta, sequenced as RSA LSA, RCCA and LCCA [8]. However instances of the LVA arising as the last branch of the aortic arch distal to the LSA are very rare and with yet undetermined incidence, not even in large sample studies. Saeed UA however reported 3 cases of aberrant RVA arising as the last branch of the aortic arch, a very rare variant [9].

Of all cases, vertebral arteries that arose from the aortic arch were much more likely to not only have a more medial course (especially their preforaminal segment) over the cervical vertebra bodies but also to enter a transverse foramen that was more cranially located than the normal C6 entrance of the vertebral artery. Spine surgeons who approach the anterior cervical spine should be aware that an aortic arch origin of the LVA is likely to be closer to the midline and less protected above the C6 vertebral level. Tardieu et al (2017) [10].

SIKKA A reported the instance of LVA which took origin from the arch of aorta and entered the foramen transversarium of the fourth cervical vertebra. While RVA took origin from the RSA close to its origin and entered the foramen transversarium of the third cervical vertebra [11]. Our reported variant like in other vertebral arteries of aortic origin showed a medial-ward coursing and less protected above C6 since this was found entering at the C5 transverse foramina.

Most aberrant vertebral artery which including those of the RVA which may arise from a trifurcating $\mathrm{BCT}$ or those arising directly from the LCCA or from the thyrocervical trunk are often asymptomatic.

The normally arising $\mathrm{R}$ or $\mathrm{L}$ vertebral artery, arising from its ipsilateral subclavian artery postero superiorly serves an important path physiological role in instances of occlusion occurring at the proximal origin of its ipsilateral subclavian artery, a path physiological state named the Subclavian Steal Syndrome (SSS).

In assuming an occlusion at the proximal origin of the LSA in the case we are reporting and a hypothetic retrograde flow in the aberrant RVA, such retrograde flow will not dump into the LSA, but into the arch of the aorta proceeding to form the descending thoracic aorta, resulting in a termed "Descending Aorta Steal Syndrome (DASS), of a therapeutic role should an occlusion occur in the arch distal to the LSA (Illustration 2).

In the typical occlusion proximally occurring in the LSA and a normally arising LVA, would result in the obstructed subclavian artery obtaining its blood supply from a retrograde flow its ipsilateral LVA, a retro grade flow derived from the origin of the basilar artery formed by the union of the 2 vertebral arteries in the caudal Pons region. This is termed the SSS as depicted in Illustration 1.

\subsection{Illustrations1}

Illustration1depicts what happens in a normally arising morphology of the aortic arch with occlusion proximally in the left subclavian artery (LSA) and a retrograde flow in the left vertebral artery (LVA)- the standard Subclavian Steal Syndrome (SSS).

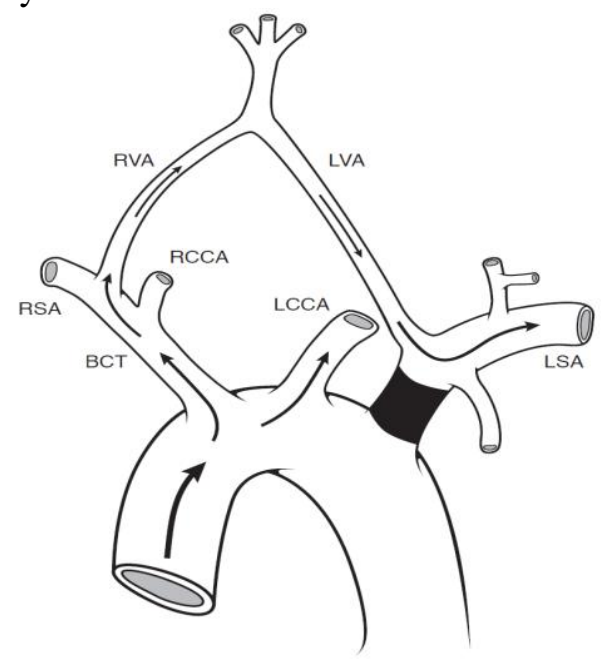

Illustration1. Image depicts the typical supra-aortic anatomy and retrograde flow in LVA when a proximal occlusion occurs in the LSA - a Subclavian Steal Syndrome (SSS). 


\subsection{Illustration2}

Illustrate 2 depicts that even in a simulated retrograde flow in the LVA that arises from the aortic arch, flow goes into the arch and not into the subclavian artery. An LVA that arises distally from the arch, like the one being reported will be beneficial when there is an occlusion in the arch distal to the left subclavian artery-producing a "Descending Aortic Steal Syndrome (DASS).

RSA; right subclavian artery, RCCA; right common carotid artery; BCT; brachiocephalic trunk, LCCA, left common carotid artery, LSA; left subclavian artery.LVA, left vertebral artery.

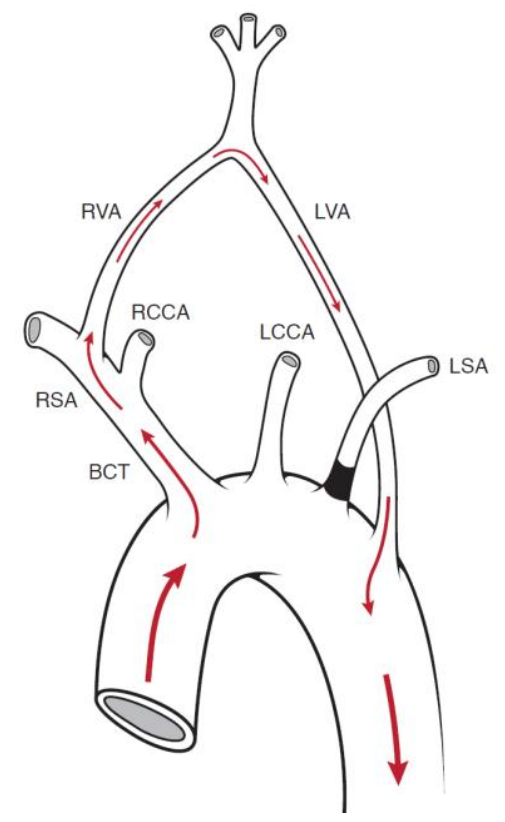

Illustration2. Hypothetic retrograde flow in LVA distal to LSA with proximal occlusion

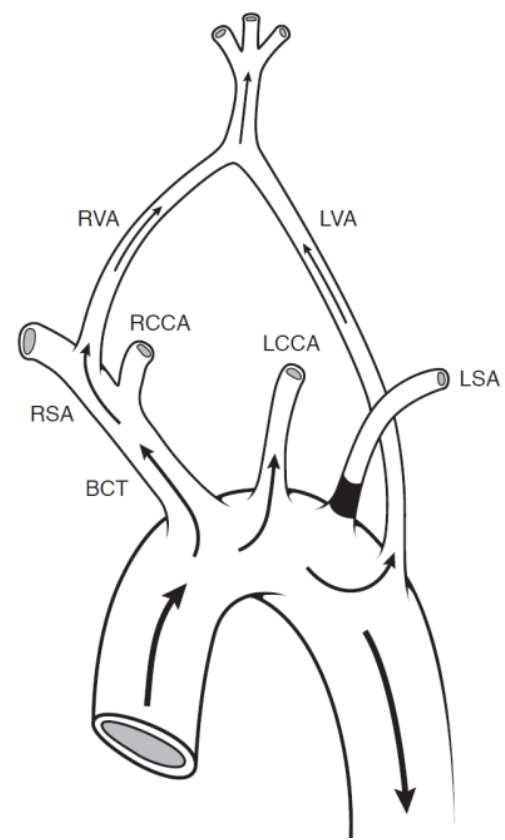

Illustration3. Normal blood flow with LVA distal to LSA with proximal occlusion

Such a retrograde flow will result in taking away some volume of blood designated for the posterior cerebral structures with a resultant postural syncope, hypo perfusion and the possibility of an ischemic stroke.

Possible management when there is an occlusion proximally without a help from a retrograde flow from the LSA will require surgical interventions including unclogging of the obstructive lesion or ligation of the variant LVA and re-anatomizing it to the LSA distal to the occlusion in the LSA.

Understanding the great vessels of the aortic arch and their variations is important for both 
the endovascular interventionist and the diagnostic radiologist. This has become more important in the era of carotid artery stents, vertebral artery stents, and new therapeutic options for intercranial interventions. Bogousslavsky J; Van Melle G, Regli F [12].

In instances of the LVA not found in its usual origin, diligent search has to be carried out to determine possible abnormal origins of this artery. In the age of increasing endovascular surgical procedures and vertebral stents, knowledge of the various aberrant origins of the VA is important in determining the different origins of the artery, its sizeand coursing so as to limit incidence of iatrogenic ligation of such aberrant LVA. Traumaticinjury to an unprotected longer than normal segment of the pre-foraminal VA due to its usually longer length may lead to an hemorrhagic stroke.

\section{ACKNOWLEDGEMENT}

Immense thanks to Prof. Stephen Peiper, sent my way to correct academic injustices, and as Chai of the Dept, encourages scholarship and providing a healthy working environment through a caring leadership.

Thanks to the Human Gift who in death still continues to contribute knowledge.

\section{REFERENCES}

[1] Jayanthi V, Prakash, Devi MN ,Grethanjali BS, Rajini T (2010).Anomalous origin of the left vertebral artery from the arch of the aorta: review of the literature and a case report. Folia Morphol (Warsz) 69 (4): 258-60.

[2] Gluncic V. Iykic G, Marin D, Percac S (1999) Anomalous origin of both vertebral arteries. Clinical Anat : 12(4) :281-4
[3] Case D, MD, Seinfeld J, MD, Folzenlogen Z, Kumpe D, MD (2015). Anomalous Right Vertebral Artery Originating from the Aortic Arch Distal to the Left Subclavian Artery: A Case Report and Review of the Literature.J .Vasc, Interv. Neurol. 8(3)21-244.

[4] Einstein EHet. al., (2016) Anomalous Origin of the Left Vertebral Artery from the Aortic Arch.Aorta (Stamford) 4(2): 64-67

[5] Shiva Kumar GL, Pamidi ,Somayaji SN, Nayak S, Volalla VR Shiva Kumar GL, Pamidi N Somayaji SN ,Nayak S, Volala V (2010) Anomalous branching pattern of the aortic arch and its clinical applications. Singapore Med J 51 (11) 3:182-183

[6] Bhatia K, Ghabriel MN, HennebergM.. Anatomical variations in the branches of the human aortic arch: a recent study of a South Australian population. Folia Morphol (Warsz). 2005; 64:217-223.

[7] Nelson ML, Sparks CD.(2001). Unusual aortic arch variation: distal origin of common carotids arteries, Clinical Anat 2001; 14: 62-5

[8] Saeed UA, Gorgos B, Semionov A, Sayegh K, Anomalous right vertebral artery arising from the arch of aorta: report of three cases. J VascInterv Neurol. 2015 Jul; 8(3): 21-24.

[9] Tardieu GG et al (2017). Aortic arch origin of the left vertebral artery: An Anatomical and Radiological Study with Significance for Avoiding Complications with Anterior Approaches to the anterior cervical spine. Published online 2012 Jun 6.

[10] Sikka A, Jain Anjali (2012).Bilateral Variation in the Origin and Course of the Vertebral Artery Anat Res Int. 580765

[11] Bogousslavsky J; Van Melle G, Regli F. The Lausanne Stroke Registry: analysis of 1,000 consecutive patients

Citation: Olutayo Ariyo. A Very Rare Origin of the Left Vertebral Artery and its Clinical Implications. ARC Journal of Cardiology. 2019; 5(2): 14-18. doi:dx.doi.org/ 10.20431/2455-5991.0502003.

Copyright: (C) 2019 Authors. This is an open-access article distributed under the terms of the Creative Commons Attribution License, which permits unrestricted use, distribution, and reproduction in any medium, provided the original author and source are credited. 